\title{
Condición física, percepción subjetiva del esfuerzo y rendimiento académico en educación primaria
}

\section{Physical fitness, rating perceived exertion and academic performance in primary school}

Víctor Ramírez Rubio; Emilio Villa González; Yahira Barranco Ruiz Universidad de Granada, (España)

Contacto: victor100439@gmail.com 


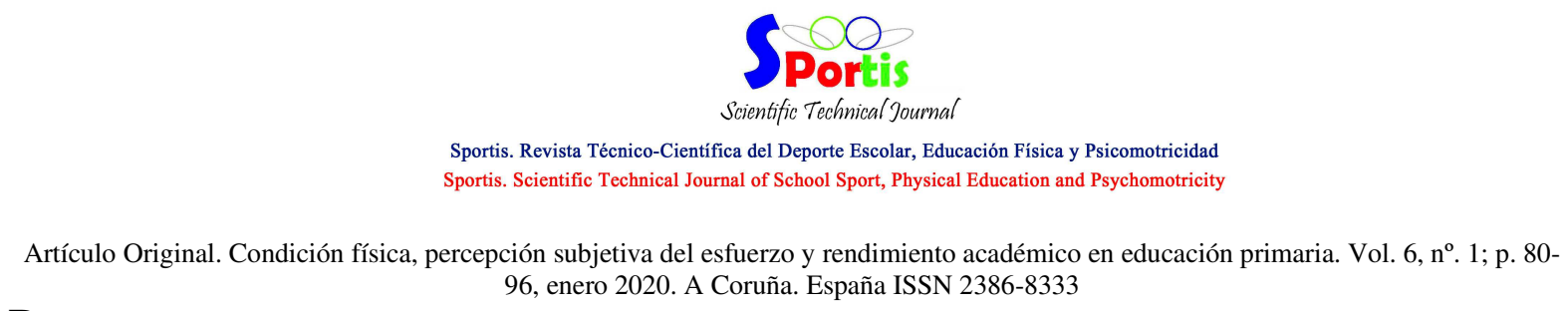

\title{
Resumen
}

Existen estudios que han conseguido establecer una relación entre el rendimiento cognitivo y académico de los alumnos y sus niveles de condición física. Los objetivos de esta investigación son estudiar la correlación existente entre el nivel de condición física y la composición corporal con el rendimiento académico, así como analizar la correlación entre dichas variables y la percepción subjetiva del esfuerzo (RPE) por género en alumnos de Educación Primaria. Un total de 69 alumnos de Educación Primaria participaron en el estudio (edad $=12,33 \pm 0,47$ años, $55,9 \%$ niñas y $44,1 \%$ niños). La condición física y la composición corporal se evaluaron siguiendo los test de la ALPHA Fitness test Battery para niños y adolescentes. El rendimiento académico se estudió mediante el promedio de notas de las asignaturas instrumentales de Educación Primaria. La RPE se registró en las clases de EF y actividades físicas escolares mediante el uso de la escala RPE de Borg (0-10). Se establecieron correlaciones significativas entre la RPE y el peso de los chicos $(\mathrm{r}=0,406, \mathrm{p}=$ $0.02)$ y las chicas $(\mathrm{r}=0,643, \mathrm{p}<0.001)$ y también con respecto al IMC, chicos $(\mathrm{r}=0,508, \mathrm{p}=$ $0.004)$, chicas $(\mathrm{r}=0,621, \mathrm{p}<0.001)$. Existió una correlación negativa significativa entre el test de la Course Navette y la RPE en el caso de los niños $(r=-0,633, p<0.001)$ y de las niñas $(r=$ $-0,452, p=0.004)$. La RPE se correlacionó significativamente con los palieres del test de Course Navette (correlación inversa) y con las variables antropométricas, como son el peso y el IMC.

\section{Palabras clave}

Condición Física; Percepción Subjetiva de Esfuerzo; Índice de Masa Corporal; Rendimiento Académico,

\begin{abstract}
There are studies in which it has been possible to establish a relationship between the cognitive and academic performance of students and their levels of physical condition. The objectives of this research are to study the correlation between the physical fitness level and body composition with the academic performance, as well as to analyze the correlation between such variables and the Rating of Perceived Exertion (RPE) of elementary school students by gender. A total of 69 primary school students participated in the study (age= $12.33 \pm 0.47$ years, $55.9 \%$ girls and $44,1 \%$ boys). The physical fitness and body composition were evaluated according to the ALPHA Fitness test Battery for children and adolescents. The academic performance was studied by means of the average of notes of the instrumental subjects of primary education. The RPE was recorded in PE classes and school physical activities by using the Borg RPE scale (0-10). Significant correlations were established between the RPE and the weight of the boys $(r=0,406, p=0.02)$ and girls $(r=0,643, p<0.001)$ and also with respect to the BMI, boys $(\mathrm{r}=0,508, \mathrm{p}=0.004)$, girls $(\mathrm{r}=0,621, \mathrm{p}<0.001)$. There was a significant negative correlation between the Course Navette test and the RPE in the case of boys $(\mathrm{r}=-0,633, \mathrm{p}<0.001)$ and girls $(\mathrm{r}=-0,452, \mathrm{p}=0.004)$. The RPE correlated significantly with the stages of the Course Navette test (inverse correlation) and with the body composition variables, such as weight and BMI.
\end{abstract}

Para citar este artículo utilice la siguiente referencia: Ramírez, V.; Villa, E.; Barranco, Y. (2020). Condición física, percepción subjetiva del esfuerzo y rendimiento académico en educación primaria. Sportis Sci J, 6 (1),80-96. DOI: https://doi.org/10.17979/sportis.2020.6.1.5704 


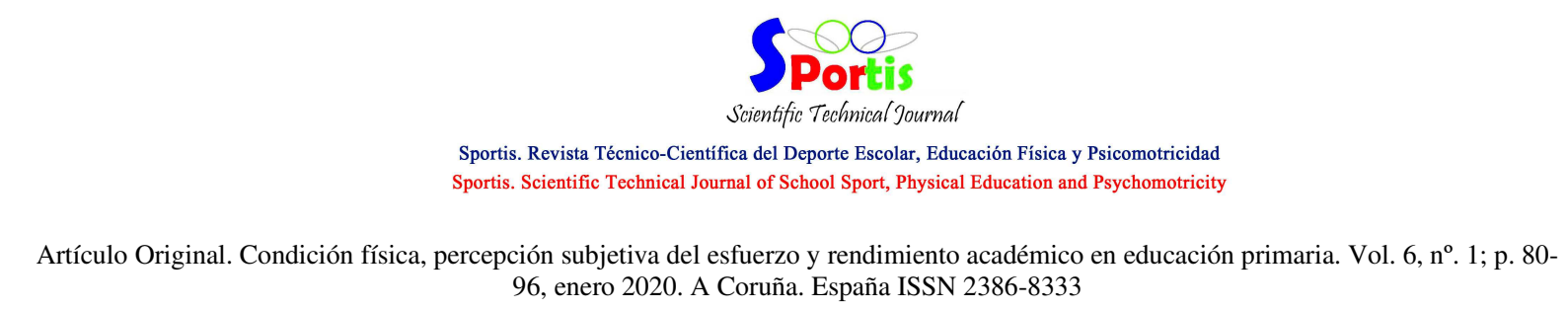

\section{Keywords}

96, enero 2020. A Coruña. España ISSN 2386-8333

Physical Fitness; Rating of Perceived Exertion; Body Mass Index; Academic Performance.

\section{Introducción}

Cuando hablamos de condición física en el ámbito educativo o escolar, buscamos encontrar el estado de forma óptimo que un niño/a debería de tener en cuanto a salud en su fase de crecimiento. Es tan importante cuidar la calidad del aprendizaje en todas las materias que se imparten en la escuela, como que los escolares desarrollen al máximo posible todas sus capacidades físicas (López, Lara, Espejo, y Cachón, 2016). Conocer la condición física del alumnado para mejorar nuestro trabajo como profesores de Educación Física, es un factor que va mas allá de simplemente impartir sesiones siguiendo unos contenidos curriculares. Para esto, buscamos jugar con la variedad en los ejercicios, la motivación de los jóvenes que presenten mayores problemas para la ejecución de ciertos juegos o tareas, conseguir una buena cooperación del grupo y por otra parte fomentar al máximo la inclusión adaptando tareas para los alumnos con alguna discapacidad, etc. (Abellán et al., 2019).

Dentro del contexto escolar, el docente de Educación Física puede influir en múltiples factores asociados al desarrollo del niño/a (psicológico, afectivo, cognitivo, sociológico, sexual y físico), incluyendo actividades físicas que pueden mejorar muchos de estas áreas y conseguir una mejora del estado de salud a nivel fisiológico, que consiga ayudar a que el alumnado mejore sus capacidades cognitivas y en consecuencia, su rendimiento académico. (Gálvez-Casas et al., 2015). La ley de Educación en España (LOMCE), expone que la finalidad educativa, es el desarrollo integral de la persona; luego si la condición física influye en todos los factores mencionados con anterioridad, los docentes de Educación Física, desde el campo de la actividad física y la salud, seremos responsables de una gran parte del desarrollo integral de los jóvenes durante la etapa escolar.

Al hilo de estas afirmaciones, existen numerosos estudios, como el del Cancela, Ayán y Sanguos (2016) en los que se ha conseguido establecer una relación entre el rendimiento cognitivo de los alumnos y sus niveles de condición física. Esto demuestra que el deporte y la actividad física benefician a los jóvenes, desde la edad escolar, mejorando sus resultados

Para citar este artículo utilice la siguiente referencia: Ramírez, V.; Villa, E.; Barranco, Y. (2020). Condición física, percepción subjetiva del esfuerzo y rendimiento académico en educación primaria. Sportis Sci J, 6 (1),80-96. DOI: https://doi.org/10.17979/sportis.2020.6.1.5704 


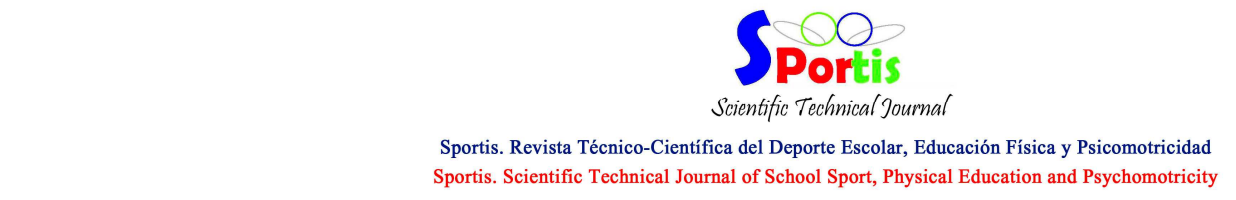

Artículo Original. Condición física, percepción subjetiva del esfuerzo y rendimiento académico en educación primaria. Vol. 6, nº $1 ;$; p. 8096, enero 2020. A Coruña. España ISSN 2386-8333

académicos. Muchos estudios señalan la relación tan estrecha que existe entre la práctica deportiva y el cambio a favor en las actitudes de muchos jóvenes, mejoras a nivel social, cognitivo, afectivo, intra- e interpersonal, refiriéndonos a este ultimo como la adquisición de valores como el respeto, compañerismo, lealtad, etc. (Beregui, 2007; Prat, 2003; Sanmartín, 2004;). Un trabajo realizado por Teixeira y Kalonoski (2003), en el que se realizaron 1000 cuestionarios a padres de niños inscritos en clubes deportivos, demuestra los diferentes motivos que tenían para inscribir a sus hijos a estos clubes y la relación que existe entre la práctica deportiva y el rendimiento escolar. Los resultados mostraban que el 85,5\% de los padres inscribían a sus hijos porque mejoraban la sociabilidad, y el resto de ellos porque querían formar atletas. En otra pregunta, el 80,9\% respondió que la escuela y el deporte, o la formación deportiva, tienen la misma importancia ya que ambos mejoraban en los jóvenes, la responsabilidad de acatar normas, trabajar en grupo y organizar el tiempo.

En general, son varios autores (Dywer, 2001; Guillamón, 2019; Linder, 1999) los que demuestran en sus investigaciones que la práctica deportiva y el rendimiento escolar tienen una relación muy positiva, apoyando la idea de que dedicar algo de tiempo a actividad física denota un incremento en los resultados académicos de los alumnos/as, sin olvidar el resto de beneficios a otros niveles antes mencionados, que también son adquiridos gracias a la práctica de deporte o actividad física.

A lo largo de estos últimos años, se ha hecho más notable la presencia del deporte durante la edad escolar, y la conciencia en los padres y madres, de que el deporte favorece la salud mental y física de sus hijos/as, es por esto que hasta las leyes educativas se están modificando para incrementar el número de sesiones de actividad física en los centros, intentando evitar caer en cifras alarmantes en enfermedades relacionadas con el sedentarismo (Solís y Borja, 2019). Por ello, los objetivos de la presente investigación fueron, en primer lugar, analizar la correlación existente entre el rendimiento académico de alumnos de educación primaria y su nivel de condición física, y en segundo lugar, analizar la correlación existente entre dicho nivel de condición física y la percepción subjetiva del esfuerzo.

\section{Método}

\section{Participantes}

Para citar este artículo utilice la siguiente referencia: Ramírez, V.; Villa, E.; Barranco, Y. (2020). Condición física, percepción subjetiva del esfuerzo y rendimiento académico en educación primaria. Sportis Sci J, 6 (1),80-96. DOI: https://doi.org/10.17979/sportis.2020.6.1.5704 


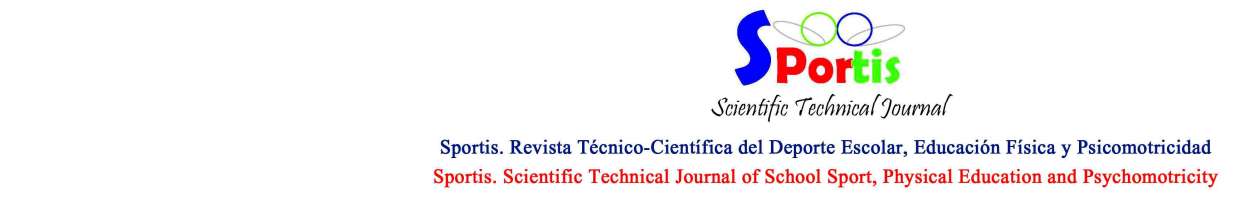

Artículo Original. Condición física, percepción subjetiva del esfuerzo y rendimiento académico en educación primaria. Vol. 6, nº $1 ;$; p. 8096, enero 2020. A Coruña. España ISSN 2386-8333

La investigación se llevó a cabo en la Ciudad Autónoma de Melilla (España). Melilla es una Ciudad Autónoma española ubicada en el noroeste costa de África, compartiendo una frontera con Marruecos. Tiene un área de $12.3 \mathrm{~km} 2$. Esta ciudad es una de las dos ciudades españolas habitadas permanentemente en África continental, además de Ceuta. Su población, según datos de 2019 es de 84.714 habitantes. El centro educativo donde se realizó la investigación fue el CEIP "Reyes Católicos", realizando dicha investigación durante 10 días aproximadamente al inicio del tercer trimestre, concretamente, durante el mes de abril del año 2017. Las actividades se realizaron en la pista polideportiva del centro escolar, para que tuvieran cabida las pruebas donde era necesario un mayor recorrido por el espacio, como por ejemplo para las pruebas de velocidad o el test de Course Navette. En el caso de las pruebas que requerían de mayor intimidad, se realizaron dentro de la sala de materiales de educación física, como son la evaluación de la composición corporal o el test de dinamometría. Antes de comenzar con el trabajo de campo se le proporcionó a la dirección escolar una carta de consentimiento informando detalladamente sobre las diferentes actividades que se iban a realizar. Fueron invitados un total de 80 jóvenes de $6^{\circ}$ de Educación Primaria por ser una edad en la que la condición física comienza a ser notable y medible, pertenecientes a un total de 3 cursos de dicho centro escolar. Por ello, la selección y reclutamiento se realizó por conveniencia, eligiendo a los participantes del presente estudio. Tras la entrega de los consentimientos, 68 alumnos/as de entre los diferentes cursos, compuestos por 30 niños y 38 niñas de entre 12 y 13 años, aceptaron participar en el estudio.

\section{Variables del estudio}

\section{Condición Física. Baterías de pruebas ALPHA Fitness}

Los niveles de condición física y la composición corporal se estudiaron mediante el uso de la Baterías de pruebas ALPHA Fitness validada en niños y adolescentes europeos como es el caso de nuestra muestra de estudio (Ruiz et al., 2011). Dicha batería de test es una de las más fiables y usada por preparadores, docentes y entrenadores del mundo del deporte para la evaluación de la condición física tanto en niños/as como adolescentes. Además, la batería de test, tiene la gran ventaja de que se realiza en un periodo de tiempo muy acotado y cualquier centro o club puede realizarla debida a su bajo coste económico.

Para citar este artículo utilice la siguiente referencia: Ramírez, V.; Villa, E.; Barranco, Y. (2020). Condición física, percepción subjetiva del esfuerzo y rendimiento académico en educación primaria. Sportis Sci J, 6 (1),80-96. DOI: https://doi.org/10.17979/sportis.2020.6.1.5704 


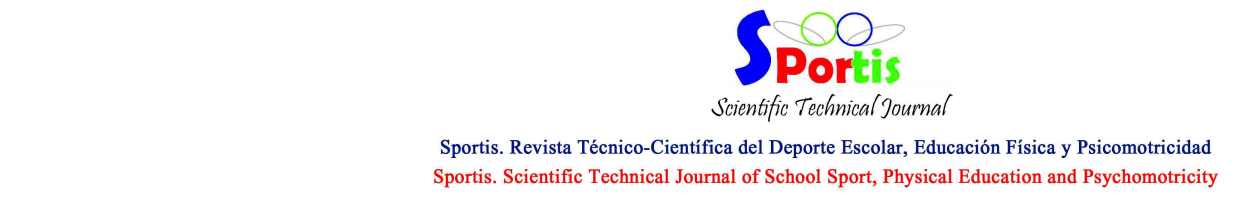

Artículo Original. Condición física, percepción subjetiva del esfuerzo y rendimiento académico en educación primaria. Vol. 6, nº $1 ;$; p. 8096, enero 2020. A Coruña. España ISSN 2386-8333

La secuencia a seguir para el análisis de variables recomendada por la batería es la siguiente: Fecha de nacimiento (edad); Peso y altura (IMC); Perímetro de la cintura; Pliegues cutáneos (tríceps y subscapular); Fuerza de presión manual (dinamometría); Salto de longitud con pies juntos; Velocidad/Agilidad 4x10m; Test de ida y vuelta de 20m (Course Navette). De todas estas variables de estudio que se recomiendan en dicha batería, en este estudio fueron evaluadas todas excepto los pliegues cutáneos, midiendo como variables de composición corporal, simplemente el peso, el IMC y el perímetro de la cintura del alumno/a. La evaluación de los test se realizó en un mismo día en horario habitual escolar de Educación Física. La duración de la aplicación de la batería fue de aproximadamente 2 horas, siendo aplicada en toda la muestra por los mismos evaluadores. Los test realizados así como las variables de estudio, se describen a continuación.

La composición corporal fue evaluada mediante las medidas de talla y altura para el cálculo del Índice de Masa Corporal (IMC) (peso corporal en kilogramos dividido por el cuadrado de la estatura en metros $(\mathrm{kg} / \mathrm{m} 2))$. También fue evaluado el perímetro de la cintura. Para ello se utilizó una báscula digital y una cinta métrica. La fuerza de los miembros superiores se midió mediante el test de prensión manual (dinamometría manual). Para ello, se utilizó el promedio de $\mathrm{Kg}$ generados tras 4 intentos de prensión manual alternados brazos, mediante el uso de un dinamómetro digital (TKK 5401 Grip D; Takey, Tokyo, Japón). La fuerza de los miembros inferiores se evaluó mediante el test de salto a pies juntos. Los participantes efectuaban 2 intentos de salto longitudinal con dos pies juntos desde una distancia establecida y en situación de parado. Las distancias ejecutadas se registraban en centímetros, considerándose para la evaluación el mejor intento. La velocidad-agilidad se evaluó mediante el test de 4x10 metros. Según el protocolo definido en la batería de pruebas ALPHA Fitness, a una distancia de 10 metros se sitúan 2 esponjas separadas a 1 metro de distancia. Durante el test, el participante debe salir a máxima velocidad a por la primera esponja y regresarla a la línea de salida, y sin pausa y siguiendo con la máxima velocidad recoger la segunda esponja y llevarla hasta la línea de partida. El participante realiza 2 intentos y se registra el mejor tiempo de los 2 intentos para la evaluación. La capacidad cardiorrespiratoria se midió mediante el test de 20 metros ida y vuelta, también conocido como el test de Course Navette. Los participantes se sitúan en una línea de partida separada de una línea de llegada por 20 metros. Mediante un

Para citar este artículo utilice la siguiente referencia: Ramírez, V.; Villa, E.; Barranco, Y. (2020). Condición física, percepción subjetiva del esfuerzo y rendimiento académico en educación primaria. Sportis Sci J, 6 (1),80-96. DOI: https://doi.org/10.17979/sportis.2020.6.1.5704 


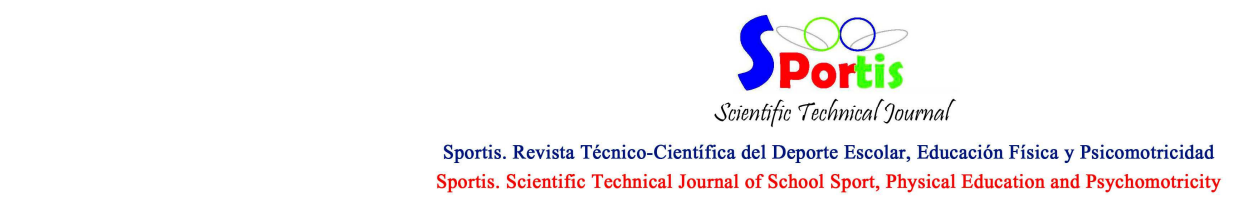

Artículo Original. Condición física, percepción subjetiva del esfuerzo y rendimiento académico en educación primaria. Vol. 6, nº $1 ;$; p. 8096, enero 2020. A Coruña. España ISSN 2386-8333

estímulo sonoro los participantes deben de desplazarse corriendo hasta la línea enfrentada y llegar antes de que el estímulo sonoro vuelva a sonar. El estímulo sonoro va aumentado su velocidad de aparición y el test finaliza cuando el participante no es capaz de seguir el ritmo o sale de las líneas de partida después de que el estímulo sonoro haya sonado.

Puede consultarse el protocolo de actuación original, descrito en detalle por Ruiz et al., (2011).

Por otra parte nos pareció interesante indagar sobre la actividad física extraescolar que los alumnos pudieran realizar, puesto que es una variable que podría estar relacionada con el nivel de condición física de los participantes. Por lo tanto, en el momento de la evaluación antropométrica, se les preguntó si realizaban algún tipo de práctica deportiva fuera del horario escolar, presentando dos opciones de respuesta, sí o no.

\section{El Rendimiento académico}

Para el análisis sobre el rendimiento académico se propusieron recoger todas las notas del $2^{\circ}$ trimestre de las asignaturas instrumentales como son las de Ciencias Sociales (CCSS), Ciencias Naturales (CCNN), Lengua Castellana y Matemáticas, ya que son estas las de más relevancia siempre en estas edades. Se realizó la media de todas ellas para buscar correlaciones significativas con el resto de variables de condición física. Los tutores de cada curso colaboraron a la hora de recoger las notas del alumnado, de modo que a pesar de que se les pidió cada una de ellas a todos los discentes de cada uno de los cursos, los tutores de los mismos, controlaron que no hubiese margen de error o falsedad en dichas puntuaciones.

\section{Percepción subjetiva del esfuerzo (RPE)}

La percepción subjetiva de esfuerzo del alumnado se evaluó en lo relativo a las sesiones de EF y las actividades físico-deportivas anexas al centro escolar que los discentes realizaron durante cada día durante el momento del estudio. Se analizaron 6 evaluaciones de RPE por alumno/a, de las cuales posteriormente se realizaría el promedio de éstas, procurando que al menos se pudieran obtener de dos unidades didácticas (UD) diferentes. En nuestro caso se han evaluado 3 sesiones de expresión corporal, y 3 de juegos pre-deportivos, diferenciando por tanto sesiones con más y menos intensidad. Se realizaron tomas de forma aleatoria para

Para citar este artículo utilice la siguiente referencia: Ramírez, V.; Villa, E.; Barranco, Y. (2020). Condición física, percepción subjetiva del esfuerzo y rendimiento académico en educación primaria. Sportis Sci J, 6 (1),80-96. DOI: https://doi.org/10.17979/sportis.2020.6.1.5704 


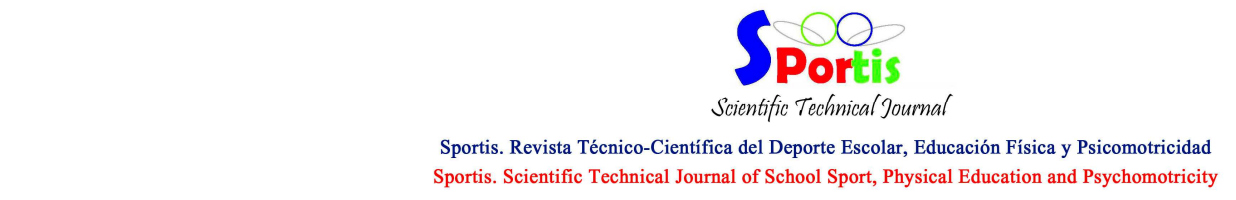

Artículo Original. Condición física, percepción subjetiva del esfuerzo y rendimiento académico en educación primaria. Vol. 6, nº $1 ;$; p. 8096, enero 2020. A Coruña. España ISSN 2386-8333

evitar que tanto el tutor de la asignatura como los alumnos/as estuvieran condicionados para ser evaluados. La metodología de análisis fue sencilla, al finalizar cada sesión se le otorga a cada alumno al llegar al aula una pequeña tabla, con una escala del 1 a 10, en la cual deben de señalar su nivel de esfuerzo percibido en la sesión que acaban de realizar. Dicha escala ya se explicó previamente para familiarizarse con ella, de modo que tengan en cuenta la importancia de su resultado y sean conscientes de que es una elección muy personal, que no se trata de una competición con el compañero de al lado, se procuró que cada uno contestase de una forma sincera y fuese crítico consigo mismo. La escala utilizada fue la escala de Borg (1982), adaptada, versión infantil, para facilitar la elección de los resultados.

\section{Análisis de datos}

Se realizaron análisis descriptivos básicos donde los resultados fueron expresados mediante la media y la desviación típica o estándar para las variables cuantitativas y mediante frecuencia y porcentajes para las variables cualitativas.

La normalidad de las variables fue estudiada mediante el test de Shapiro-Wilk. La diferencia entre las medias de las variables cuantitativas del estudio en función del sexo, se analizó mediante el test t de student de muestras independientes (variables distribución normal: composición corporal, dinamometría, test de 4x10m y RPE), y mediante el test U de Mann Whitney (variables distribución no normal: salto a pies juntos, Course Navette y rendimiento académico). La comparación estadística de las variables cualitativas en función del sexo se analizó mediante el test Chi-cuadrado. Para aquellas variables del estudio que presentaron una distribución normal, se aplicaron correlaciones bivariadas de Pearson (test paramétrico). Para las variables que presentaron una distribución no normal se aplicaron correlaciones bivariadas de Spearman (test no paramétrico).

El valor de $\mathrm{p}$ se ajusto a $\mathrm{p}<0.05$. Todos los análisis se realizaron mediante el programa IBM SPSS Statistics Compilation 1.0.0.1012 edición de 64 bits.

\section{Resultados}

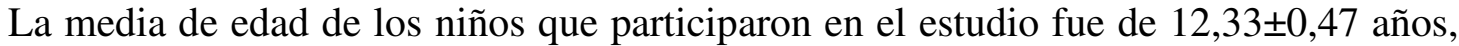
mientras que en el caso de las chicas fue de 12,16 $\pm 0,37$ años. 
Artículo Original. Condición física, percepción subjetiva del esfuerzo y rendimiento académico en educación primaria. Vol. 6, nº $1 ;$; p. 8096, enero 2020. A Coruña. España ISSN 2386-8333

En la tabla 1 se puede observar que las variables de estudio comparadas por sexo. Con respecto a las variables de composición corporal analizadas, existieron diferencias significativas entre sexos en las variables de edad y altura, alcanzando valores significativamente mayores en el grupo de los niños. En relación a las variables de condición física estudiadas, sólo en el test de salto a pies juntos, los niños presentaron un valor promedio significativamente mayor con respecto a las niñas, al igual que los niños presentaron una mayor prevalencia en relación a la práctica habitual de algún deporte. Por lo contrario, fueron las niñas las que presentaron notas promedio significativamente mayores en todas las asignaturas analizadas con respecto a las presentadas por los niños.

Tabla 1. Características descriptivas de las variables de estudio por sexo.

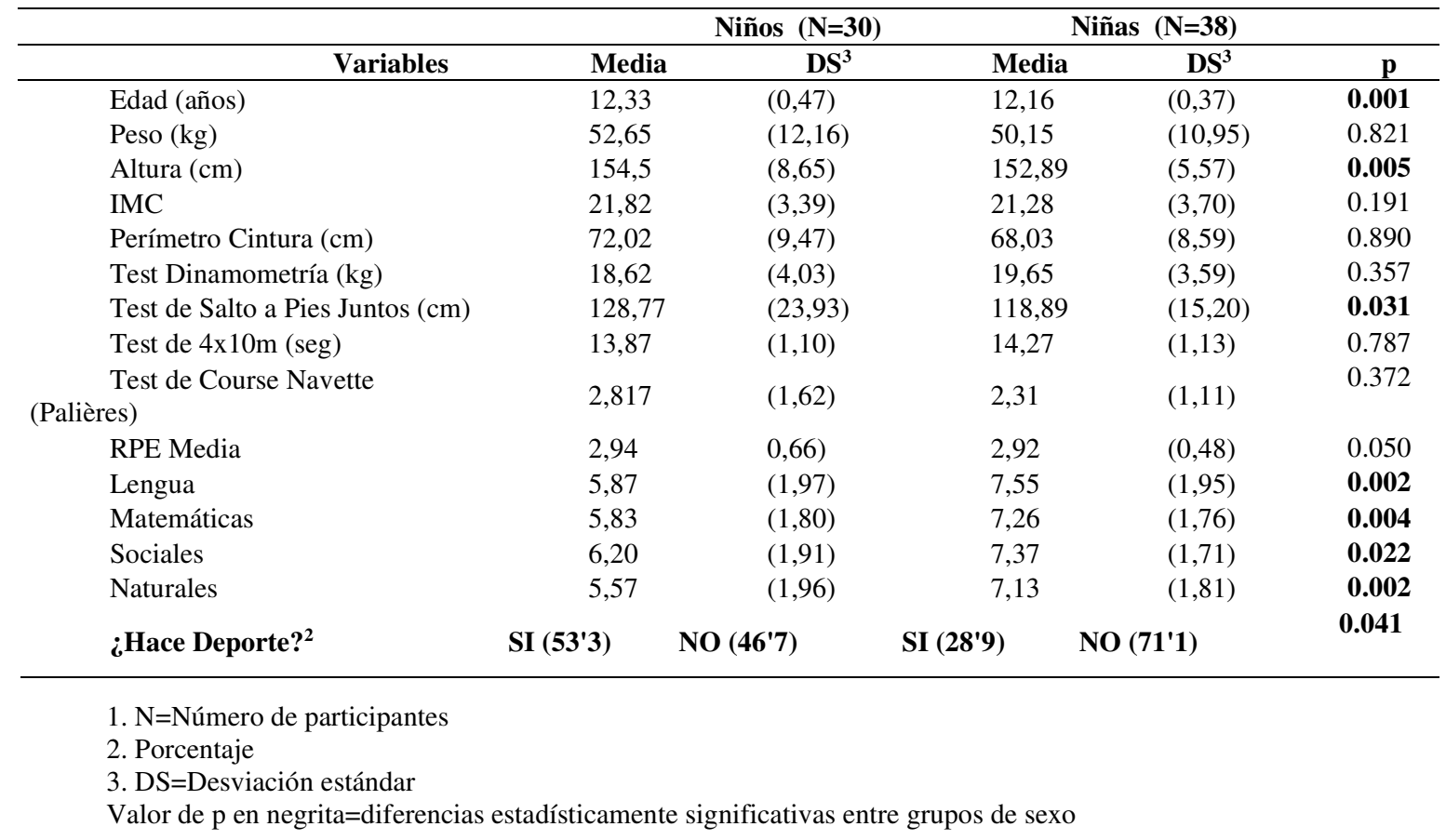

A continuación se establecen las correlaciones entre la RPE y las notas medias académicas con las variables de composición corporal y las de condición física respectivamente. (Ver tabla 2). 


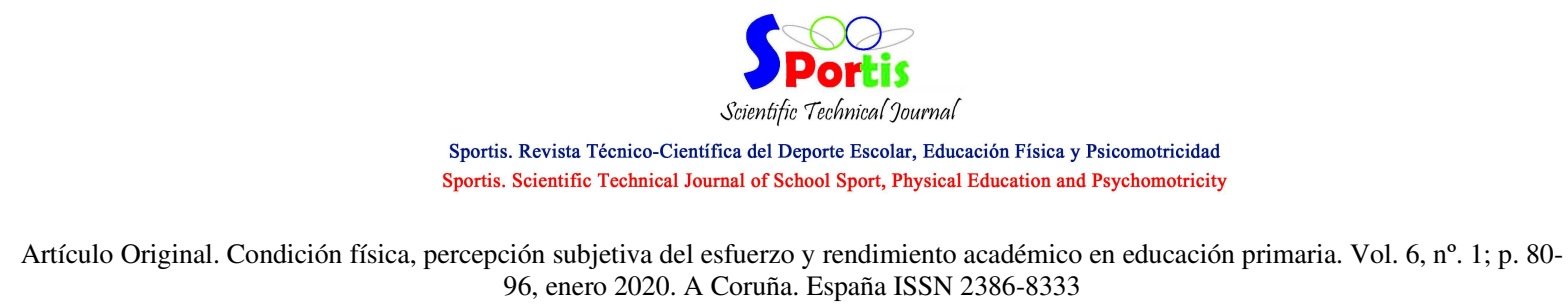

Tabla 2. Correlaciones de la RPE y las notas académicas con las variables de estudio discriminadas por sexo

\begin{tabular}{|c|c|c|c|c|}
\hline & \multicolumn{2}{|c|}{ RPE (media) } & \multicolumn{2}{|c|}{ Notas Académicas (media) } \\
\hline & Niños & Niñas & Niños & Niñas \\
\hline \multicolumn{5}{|l|}{ Composición corporal } \\
\hline Peso $(\mathrm{Kg})$ & $0,406^{*}$ & $0,643 *$ & $-0,037$ & $-0,300$ \\
\hline Altura (cm) & 0,129 & $0,470^{*}$ & $-0,055$ & $-0,130$ \\
\hline $\operatorname{IMC}\left(\mathrm{Kg} / \mathrm{m}^{2}\right)$ & 0,508* & $0,621 *$ & $-0,016$ & $-0,321^{*}$ \\
\hline Perímetro Cintura (cm) & $0,455^{*}$ & $0,570 *$ & $-0,020$ & $-0,249$ \\
\hline \multicolumn{5}{|l|}{ Condición Física } \\
\hline Dinamometría (kg) & 0,064 & 0,161 & $-0,087$ & $-0,112$ \\
\hline Test de Salto a Pies Juntos (cm) & $-0,277$ & $-0,429 *$ & $-0,267$ & $-0,243$ \\
\hline Test de 4x10m (seg) & $0,392 *$ & 0,051 & $-0,204$ & $-0,084$ \\
\hline Test de Course Navette (Palières) & $-0,633 *$ & $-0,452 *$ & $-0,085$ & 0,215 \\
\hline
\end{tabular}

* Coeficiente de correlación estadísticamente significativo $(\mathrm{p}<0,05)$.

IMC: índice de masa corporal

A simple vista se pudo observar que existía correlación entre la RPE y algunas variables, principalmente de composición corporal. Se observó una correlación y moderada entre el peso y la RPE media, esto significa que los alumnos que tienen un peso mayor son aquellos que reportan una mayor RPE, tanto en niños $(p=0.02)$ como niñas $(p<0.001)$. Las niñas, presentaron una correlación significativa, entre RPE y altura $(p=0.03)$. El IMC proporcionó datos más relevantes puesto que existen valores correlacionales mayores a $\mathrm{r}=$ 0,50. En este caso ambos sexos mantuvieron una gran relación entre las variables RPE media reportada y su IMC (niños, p= 0.004; niñas, p< 0.001), indicando esta relación que un sujeto con un mayor IMC por norma general declara también una RPE mayor. Bajo esta relación se observó que son el colectivo de las féminas las que arrojan valores más significativos. Los valores para la circunferencia abdominal que reportaron ambos sexos también demostraron tener una correlación moderada y muy significativa (niños, $\mathrm{p}=0.01$; niñas, $\mathrm{p}<0.001$ ). Las cifras sobre el perímetro de la cintura en relación a la RPE Media mostraban, al igual que en la anterior correlación, que son las niñas las que, a mayor perímetro, mayor es la RPE 


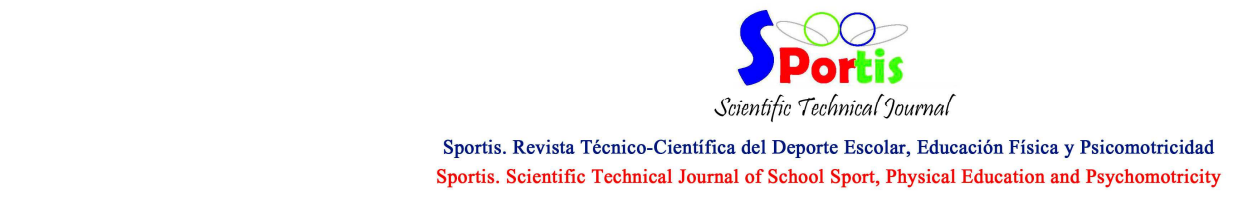

Artículo Original. Condición física, percepción subjetiva del esfuerzo y rendimiento académico en educación primaria. Vol. 6, nº $1 ;$; p. 8096, enero 2020. A Coruña. España ISSN 2386-8333

recogida. Por último, el mayor valor de correlación apareció en la relación entre los palieres conseguidos en el test de Course Navette y la RPE. Se trata de un resultado negativo puesto que dicha correlación es inversa, de modo que cuantos más palieres conseguidos en el test de ida y vuelta de $20 \mathrm{~m}$, menor es la RPE reportada. Con respecto a los niños y niñas fue el grupo varón quien presentó una mayor correlación entre dichas variables de análisis (niños, p= 0.001; niñas, $\mathrm{p}=0.004)$.

En relación a las notas académicas, no se observaron correlaciones significativas para ninguna variable. Se produjo una correlación muy leve con el IMC y solo exclusivamente en las niñas $(\mathrm{p}=0.05)$.

\section{Discusión}

Los principales hallazgos de esta investigación arrojan que no existen correlaciones significativas entre el rendimiento académico de los alumnos de Educación Primaria del estudio y su nivel de condición física. Sin embargo, cuando se estudió la relación entre los test de condición física y composición corporal de la batería de pruebas ALPHA Fitness, y la RPE reportada en las prácticas deportivas y de actividad física realizadas en el contexto escolar, sí se encontraron correlaciones significativas. El IMC y el test de Course Navette que evalúa la capacidad cardiorrespiratoria, mostraron correlaciones significativas fuertes (correlación positiva y correlación inversa, respectivamente) en ambos sexos de la muestra de estudio.

La relación entre la RPE media reportada por ambos sexos y el índice de masa corporal demostró que encontrarse en unos valores óptimos de IMC puede influir en la fatiga, puesto que los niños que tienen este índice más elevado reportaron una mayor percepción subjetiva del esfuerzo. Si el IMC está directamente relacionado con un buen estado de forma y de salud, es totalmente comprensible que a medida que suben estos niveles, suban de manera proporcional los niveles de fatiga al realizar un esfuerzo, mejor estado de forma, menor fatiga o presentación de la fatiga más tardía. Se estima que presentar un IMC entre 2025 está relacionado con un riesgo de mortalidad bajo y por lo tanto, son niveles de composición corporal saludables, mientras que con valores superiores a $25 \mathrm{~kg} / \mathrm{m}^{2}$ se comienza a hablar de sobrepeso, obesidad y problemas realmente alarmantes para la salud en estas

Para citar este artículo utilice la siguiente referencia: Ramírez, V.; Villa, E.; Barranco, Y. (2020). Condición física, percepción subjetiva del esfuerzo y rendimiento académico en educación primaria. Sportis Sci J, 6 (1),80-96. DOI: https://doi.org/10.17979/sportis.2020.6.1.5704 
Artículo Original. Condición física, percepción subjetiva del esfuerzo y rendimiento académico en educación primaria. Vol. 6, nº $1 ;$; p. 8096, enero 2020. A Coruña. España ISSN 2386-8333

edades. En un estudio realizado a jóvenes universitarios de la universidad de Chile (Duran, Valdés, Godoy, y Herrera, 2014), donde un 35,6\% de 239 participantes presentaron un IMC categorizado como obesidad o sobrepeso, puso de manifiesto que el hecho de una mala alimentación y baja actividad física, y en consecuencia, un aumento del IMC, se relacionaba con peores resultados en el test de capacidad cardiorrespiratoria de Course Navette.

Si bien uno de nuestros objetivos era buscar si existía una relación entre las variables de condición física y la RPE, los resultados de la RPE Media obtenidos en la presente investigación son bastante bajos, entre 2 y 3 en la escala de Borg. Según dicha escala los escolares asocian las sesiones de educación física a un esfuerzo moderado. Lo que puede dar lugar a pensar 2 hipótesis: o los niños/as presentaban aburrimiento y falta de interés en las sesiones, o por el contrario las unidades didácticas expuestas, a pesar de que los alumnos/as se exigieron al nivel que deberían, no son de una intensidad tan alta como para que un niño en educación primaria reporte unos valores alarmantes en dicha escala. Una de las correlaciones más interesantes en este trabajo fue la relación inversa entre la percepción subjetiva del esfuerzo de los niños, con los palieres conseguidos en el test de capacidad cardiorrespiratoria de Course Navette. Esto afirma lo expuesto en los puntos anteriores de esta investigación, haciendo alusión a que existe correlación entre la RPE y la capacidad cardiorrespiratoria. Por tanto, parece que la RPE podría ser un una buena herramienta para controlar la intensidad cardiovascular en las sesiones de EF, tanto a nivel colectivo como individual. En un estudio realizado por Cuadrado (2010), donde fueron evaluados 13 sujetos de un equipo de balonmano de la División de Honor B española de su frecuencia cardiaca máxima (FC máx.) y su consumo máximo de oxigeno $\left(\mathrm{VO}_{2}\right.$ máx.) mediante el test de Course Navette, y de los valores de RPE reportados durante 21 sesiones de trabajo, se estudió posteriormente, la relación entre los niveles de condición cardiorrespiratoria y los valores de RPE reportados. Los valores correlacionales adquiridos fueron de $\mathrm{r}=0,88$ mostrando una correlación fuerte entre ambas variables. A diferencia de nuestro estudio, aquí se pudo evaluar la RPE durante un periodo mayor de tiempo, y en dos tipos, individual y colectivo. A pesar de que fuesen a deportistas de 16 años y nuestro estudio sea con niños/as de 12/13, queda claro la importancia de medir la percepción subjetiva de esfuerzo en cualquier ámbito, ya sea deportivo o escolar, 


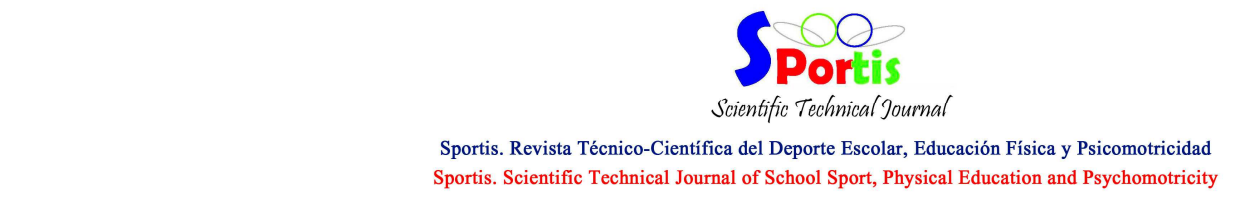

Artículo Original. Condición física, percepción subjetiva del esfuerzo y rendimiento académico en educación primaria. Vol. 6, nº $1 ;$; p. 8096, enero 2020. A Coruña. España ISSN 2386-8333

conociendo además que presenta correlaciones significativas para alguno de los valores de condición física.

Con respecto al rendimiento académico escolar, las nota promedio de las asignaturas instrumentales de los niños/as de la provincia de Melilla no son demasiado altas. El informe PISA en los últimos años no ha introducido datos de la comunidad autónoma, y es que esta ciudad soporta uno de los mayores porcentajes de fracaso o abandono escolar. En esta investigación se quiso valorar solo las asignaturas instrumentales por ser estas a las que más importancia se les otorga en Educación Primaria, como lo son Lengua Castellana, Matemáticas, Ciencias Sociales y Ciencias Naturales. El resultado fue un promedio de notas inferior a notable para los 68 participantes del estudio, siendo las niñas, las que mostraron un valor promedio de nota más alto que los niños en estas asignaturas. Dichos resultados, coindicen con otro estudio similar (Castro, Pérez, Cachón, y Zagaláz, 2016) en el que el sexo es el diferenciador principal de las calificaciones de estas materias. En Canadá, se realizó un estudio a más de 5000 sujetos de 11 años, donde se analizó si el realizar actividad física es determinante en el rendimiento escolar (Tremblay, Inman, \& Willms, 2000). Los resultados fueron negativos, los cuales expresaron que los alumnos que realizaban más actividad física eran los que peores notas sacaron. Por otro lado si existen estudios en los cuales los alumnos que mejores resultados obtuvieron en test basados en la capacidad cardiorrespiratoria como el test de Course Navette, eran aquellos discentes que mejor rendimiento escolar mostraban. (Pertusa, Sanz-Frías, Salinero, Pérez-González y García-Pastor, 2018) En nuestro estudio, a pesar de que son los niños los que parece que realizan algo más de deporte en horario extraescolar, son también ellos los que reportan una media inferior en sus expedientes académicos.

\section{Limitaciones de estudio}

La presente investigación tiene una serie de limitaciones que se deben de declarar. La ciudad donde se ha realizado la investigación presenta una serie de factores tanto culturales, como socioeconómicos que podrían tener importancia de cara a los resultados principales del estudio (Estrada, Ma.M., 2017). Dichos factores, no han sido evaluados en la presente investigación, por lo que necesitarían nuevas investigaciones que abarquen la incidencia de

Para citar este artículo utilice la siguiente referencia: Ramírez, V.; Villa, E.; Barranco, Y. (2020). Condición física, percepción subjetiva del esfuerzo y rendimiento académico en educación primaria. Sportis Sci J, 6 (1),80-96. DOI: https://doi.org/10.17979/sportis.2020.6.1.5704 
Artículo Original. Condición física, percepción subjetiva del esfuerzo y rendimiento académico en educación primaria. Vol. 6, nº . 1; p. 8096, enero 2020. A Coruña. España ISSN 2386-8333

dichos factores sociodemográficos sobre esta relación entre el nivel de condición física y el rendimiento académico en alumnos de Educación Primaria. Por otro lado, no hemos evaluado de manera objetiva los niveles de actividad física mediante acelerometría u otras técnicas de evaluación propuestas en la literatura, sino que evaluamos esta variable mediante una pregunta auto-reportada, pudiendo esto aumentar el sesgo de evaluación, aunque ésta no fue una variable principal de estudio. Sin embargo, no contábamos con suficientes medios económicos ni logísticos para llevar a cabo dicha evaluación. Se recomienda dicha evaluación objetiva en futuras investigaciones.
\end{abstract}

\title{
Conclusiones
}

Como conclusiones, podemos decir que existe una correlación media-alta entre las variables de composición corporal, peso e IMC, y la RPE, así como una correlación inversa entre la capacidad cardiorrespiratoria medida mediante el test de Course Navette y la RPE para ambos sexos de la muestra de estudio.

Con respecto al rendimiento académico y el nivel de condición física de los participantes del estudio, no existieron correlaciones significativas. Como se ha comentado en el apartado anterior, influyen múltiples factores ajenos al centro escolar que determinan las calificaciones escolares de los discentes, por lo que nuevas investigaciones que analicen la influencia de dichos factores en esta relación deben ser estudiados ampliamente en un futuro.

Como aplicación práctica derivada de este estudio, se recomienda practicar y entrenar previamente la RPE mediante el uso de la escala de Borg (0-10) con el alumnado, para asegurar un registro y control optimizado de la intensidad cardiovascular de las sesiones de Educación Física en Educación Primaria. Además, sería interesante realizar dicho estudio comparando diferentes tipos de intensidad durante las sesiones.

\section{Referencias}

Abellán, J., Sáez, N. Mª, Reina, R., Ferriz, R., y Navarro, R. (2019). Percepción de autoeficacia hacia la inclusión de futuros maestros de educación física. Revista de Psicología del Deporte 28 (2) 134-146. Recuperado de: https://www.researchgate.net/profile/Ruben_Navarro_Paton/publication/330567114_Percepci 
Artículo Original. Condición física, percepción subjetiva del esfuerzo y rendimiento académico en educación primaria. Vol. 6, nº 1; p. 8096, enero 2020. A Coruña. España ISSN 2386-8333

on_de_autoecacia_hacia_la_inclusion_en_futuros_maestros_de_educacion_fisica/links/5c48d 47392851c22a38c1243/Percepcion-de-autoecacia-hacia-la-inclusion-en-futuros-maestros-deeducacion-fisica.pdf

Beregüi, R., Garcés de los Fayos, E. (2007). Valores en el deporte escolar: estudio con profesores de educación física. Cuadernos de Psicología del Deporte 7 (2) 89-104. Recuperado de: http://revistas.um.es/cpd/article/view/54621

Brito, Mㄹ., Ruiz, J.A., Navarro, M., García J.M. (2009). Valoración de la condición física y biológica en escolares. Sevilla: Wanceulen editorial deportiva.

Cancela, J.M., Ayan, C., Sanguos, M.J. (2016) Relación entre la condición física y rendimiento académico en matemáticas y lenguaje en estudiantes españoles en educación secundaria: un estudio longitudinal. CCD. Cultura_Ciencia_Deporte 11 (31) 7-16 Recuperado de: http://ccd.ucam.edu/index.php/revista/article/view/638 DOI: https://doi.org/10.12800/ccd.v11i31.638

Castro, R., Pérez, V. Cachón, J., Zagalaz, Mª.L. (2016) Valoración de la relación entre rendimiento académico y condición física en escolares. Revista Euroamericana de Ciencias del Deporte 5 (1) 47-54. Recuperado de: https://revistas.um.es/sportk/article/download/249111/189371/0 DOI: https://doi.org/10.6018/249111

Cuadrado, J. (2010). Análisis de la influencia de la intensidad del entrenamiento sobre variables de control de la carga interna en deportes colectivos. (Tesis doctoral, Universidad de Granada). Recuperado de http://digibug.ugr.es/handle/10481/5574

Duran, S., Valdés, P., Godoy, A. y Herrera, T. (2014) Hábitos alimentarios y condición física en estudiantes de pedagogía en educación física. Revista chilena de nutrición. 43 (3) 251-259. DOI: https://doi.org/10.4067/S0717-75182014000300004

Dwyer, T., Sallis, J. F., Brizar, L., Lazarus, R. \& Deán, K. (2001). Relation of Academic Performance to Physical Activity and Fitness in Children. Pediatric Exercise Science, 13, 225-238. Retrieved from www.sparkpe.org/wpcontent/uploads/2010/03/Relation-of-Academic-Performance-to-P-A-and-Fitness-inChildren.pdf DOI: https://doi.org/10.1123/pes.13.3.225 


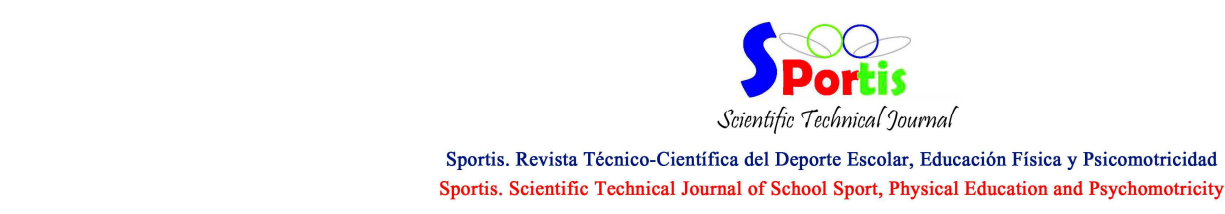

Artículo Original. Condición física, percepción subjetiva del esfuerzo y rendimiento académico en educación primaria. Vol. 6, nº 1; p. 8096, enero 2020. A Coruña. España ISSN 2386-8333

Estrada, Mª.M. (2017) Estudio descriptivo sobre el abandono escolar temprano e influjo de variables personales y socio-culturales en la ciudad autónoma de Melilla. (Tesis doctoral, Universidad de Granada). Recuperado de: http://digibug.ugr.es/handle/10481/46812

Gálvez-Casas, A., Rodríguez García, P. L., García-Cantó, E., Rosa Guillamón, A., Pérez-Soto, J. J., Marcos, L. T., y López, P. T. (2015). Capacidad aeróbica y calidad de vida en escolares de 8 a 12 años. Clínica e Investigación en Arteriosclerosis, 27(5), 239-245. DOI: https://doi.org/10.1016/j.arteri.2015.01.001

Guillamón, A. R., Canto, E. G., y López, P. J. C. (2019). Capacidad aeróbica y rendimiento académico en escolares de educación primaria. Retos: nuevas tendencias en educación física, deporte y recreación, (35), 351-354.

Linder, K. (1999). Sport Participation and Perceived Academic Performance of School Children and Youth. Pediatric Exercise Science, 11, 129-144. DOI: https://doi.org/10.1123/pes.11.2.129

López, F.J., Lara, A.J., Espejo, N. y Cachón, J. (2016) Influencia del género la edad y el nivel de actividad física en la condición física de alumnos de educación primaria. Revisión bibliográfica. Retos: nuevas tendencias de educación física, deporte y recreación. 29 129-133 Recuperado de: https://dialnet.unirioja.es/servlet/articulo?codigo=5400858

Pertusa, G., Sanz-Frías, D., Salinero, J. J., Pérez-González, B., y García-Pastor, T. (2018). Rendimiento académico y su relación con niveles de actividad física y de condición física en adolescentes. Revista de psicología del deporte, 27(1), 125-130.

Prat, G., \& Prat, S. (2003). Actitudes valores y normas en educación física y el deporte, Barcelona, España, INDE Publicaciones

Ruiz, J. R., España Romero, V., Castro Piñero, J., Artero, E. G., Ortega, F. B., Cuenca García, M., Castillo, M. J. (2011). Batería ALPHA-Fitness: test de campo para la evaluación de la condición física relacionada con la salud en niños y adolescentes. Nutrición Hospitalaria, 26(6). Recuperado

de http://scielo.isciii.es/pdf/nh/v26n6/03_articulo_especial_02a.pdf

Sanmartín, M. G. (2004). El valor del deporte en la educación integral del ser humano. Revista de educación, 335, 105-126. Recuperado de: http://www.revistaeducacion.educacion.es/re335/re335_10.pdf 
Artículo Original. Condición física, percepción subjetiva del esfuerzo y rendimiento académico en educación primaria. Vol. 6, nº $1 ;$; p. 8096, enero 2020. A Coruña. España ISSN 2386-8333

Solís, P., Borja, V. (2019). Niveles de actividad física y sedentarismo en escolares de $3^{\mathrm{o}}$ y $4^{\mathrm{o}}$ de educación primaria. EmasF: revista digital de educación física, (56), 119-131. Recuperado de: https://dialnet.unirioja.es/servlet/articulo?codigo=6751147

Teixeira, C., Kalinoski, S. (2003). La importancia del deporte como factor social en las matrículas en escuelas deportivas de la administración pública de Pindamonhangaba, Brasil. Efdeportes, Revista Digital 9 (60). Recuperado de http://www.efdeportes.com/efd60/social.htm

Tremblay, M.S., Inman, J.W. Willms J.D. (2000) The relationship between physical activity, self-esteem, and academic achievement in 12-year-old children. Pediatric Exercise Science. 12 312-23 DOI: https://doi.org/10.1123/pes.12.3.312 